\title{
Estudio taxonómico del género Paraxeropsis Camousseight, 2008 (Insecta, Phasmatodea), con la descripción de $P$. camousseighti n. sp.
}

\section{Taxonomics of the genus Paraxeropsis Camousseight, 2008 (Insecta, Phasmatodea), with description of $P$. camousseighti sp. $\mathrm{n}$.}

\author{
Alejandro Vera ${ }^{1}$ \\ ${ }^{1}$ Universidad Metropolitana de Ciencias de la Educación, Departamento de Biología, Facultad de Ciencias Básicas, Av. José \\ Pedro Alessandri 774, Ñuñoa, Santiago, Chile. \\ E-mail: alveras2@gmail.com.
}

\begin{abstract}
RESUMEN
Se describen los imagos de ambos sexos y el huevo de Paraxeropsis camousseighti n. sp., además se entregan observaciones biológicas, sobre la base de especímenes colectados en Chile, en una única localidad de la costa de la Región del Maule. $\mathrm{Su}$ descubrimiento permite complementar la diagnosis original del género, discutir la morfología genital y compararla con otras especies de fásmidos recientemente estudiadas.
\end{abstract}

Palabras clave: Chile, Heteronemiidae, morfología, taxonomía.

\begin{abstract}
The imagos of both sexes and the egg of Paraxeropsis camousseighti sp. n. are described, as well as biological observations of specimens collected in Chile in one locality near the coast in the Región del Maule. Its discovery complements the original diagnostic description of the genus and allows discussion of genital morphology and comparison with other recently studied species of phasmids.
\end{abstract}

KEYwords: Chile, Heteronemiidae, morphology, taxonomy.

\section{INTRODUCCIÓN}

La familia Heteronemiidae (sensu Zompro 2004) está representada en Chile por un conjunto de 6 géneros: Heteronemia Gray 1835, Minteronemia Zompro 2004, Splendidonemia Zompro 2004, Spinonemia Zompro 2004, Xeropsis Redtenbacher 1906 y Paraxeropsis Camousseight 2008, estos cuatro últimos de carácter monoespecífico.

Los trabajos de Gray (1835), Brunner \& Redtenbacher (1906-1908) y Zompro (2004) examinan los rasgos de morfología externa que clásicamente han sido utilizados para establecer los taxa. Algunos de éstos, como la coloración, las foliaciones y excrecencias tegumentarias pueden presentar gran variabilidad ya que en su mayoría se relacionan con elementos del camuflaje. Esta variabilidad ha hecho necesaria la evaluación posterior de las descripciones originales, mediante la incorporación de nuevos especímenes y el estudio de nuevos rasgos. Sin embargo, los hábitos crípticos de estos insectos los hacen poco frecuentes en las colecciones y el examen de los taxa puede tardar mucho en espera de nuevos individuos (Camousseight 1995).

El estudio morfológico de los huevos (Sellick 1997, 2000; Camousseight \& Bustamante 1991), así como el de la morfología genital de ambos sexos, y muy especialmente de sus partes blandas, ha resultado mucho más informativo de lo que había demostrado ser la morfología externa tradicional. Los sucesivos trabajos en este tema (Camousseight 1986, 1988, 2008; Camousseight \& Vera 2011) permiten reconocer en estas estructuras un robusto valor de diagnosis para cada taxón estudiado.

En esta oportunidad se ha examinado una pareja de 
especímenes colectados manualmente durante la noche, los que fueron mantenidos vivos por varios días para recuperar un número adecuado de huevos. Tanto la morfología de los huevos como la de los imagos evidenciaban un nuevo taxón, el que fácilmente podría haber sido atribuido a una nueva categoría genérica; sin embargo, la morfología genital ha permitido atribuirlo sin error a Paraxeropsis. Desde su hallazgo, se ha intentado infructuosamente reunir especímenes adicionales, colectando en repetidas oportunidades y con variados métodos de muestreo en la localidad de origen. De este modo se propone la nueva especie, entregando la descripción de ambos sexos y los huevos sobre la base de especímenes únicos.

\section{MATERIALES Y MÉTODOS}

Los especímenes están conservados en etanol y depositados en la colección entomológica del Museo Nacional de Historia Natural de Santiago de Chile (MNHN). Se estudiaron un macho, una hembra y 26 huevos, los datos de colecta se indican junto a la descripción de la especie. Los especímenes fueron colectados manualmente sobre Chusquea sp. y Blechnum chilense (Kaulf) a orillas de un estero, próximo a la Reserva Natural Los Ruiles ( $35^{\circ} 49^{\prime} \mathrm{S} / 72^{\circ} 38^{\prime} \mathrm{W}$ ), durante un muestreo nocturno, iluminando el follaje del sotobosque con una lámpara. La pareja fue conservada viva durante 13 días, período en que la hembra ovipuso 26 huevos. Las genitalias fueron aclaradas con $\mathrm{KOH}$ al $10 \%$ durante $24 \mathrm{hrs}$ e inyectadas con agua para su expansión, fijadas en alcohol de $70 \%$ y teñidas con negro de clorazol. La descripción de los huevos sigue a Sellick 1997 y la de los imagos a Camousseight 1986, 2008 y Camousseight \& Vera 2011.

Simbología utilizada en las figuras: $B c a=$ bursa copulatrix, porción anterior o basal; $B c p=$ bursa copulatrix, porción posterior y apical; $C=\operatorname{cercos} ; D v=$ divertículos; $E s f=$ espermatóforo; $E s p=$ espermateca; $G=$ gonapófisis; $L u 1=$ lóbulos genitales masculinos, unidad $1, L u 3=$ unidad 3, $L u 4=$ unidad 4, Lu5= unidad 5; Od= oviducto; $O v=$ ovipositor; $P s g=$ placa subgenital; Resp= ramificaciones de la espermateca; $V=$ vómer; $V d=$ valva (= Gonapófisis) superior o dorsal; $V m=$ valva media; $V i=$ valva inferior $o$ ventral; VII= séptimo segmento abdominal; VIII= octavo segmento abdominal; $I X=$ noveno segmento abdominal; $X=$ décimo segmento abdominal; $X s p=$ espinas marginales del décimo segmento abdominal.

\section{RESULTADOS}

Género Paraxeropsis Camousseight, 2008

Paraxeropsis Camousseight, 2008: 109. Especie tipo: Paraxeropsis bicristata Camousseight 2008, por designación original. Holotipo depositado en MNHN N:6799.

Diagnosis: Macho más delgado que la hembra, con $2 / 3$ de la longitud corporal de la hembra. Ambos sexos ápteros. Cuerpo con gránulos de tamaño variable, tanto en los esternos como en los tergos. Cabeza, oblonga. Tórax: protórax, más largo que ancho; mesotórax, 3 a 4 veces más largo que el protórax; metatórax y segmento medio de talla semejante; tibias medias y posteriores con area apicalis. Abdomen con segmento anal de margen posterior bilobulado, en la hembra inclinado ventralmente y en el macho proyectado distalmente.

Genitalia: Hembra con lamina subgenital cóncava, de margen posterior coincidente o apenas sobrepasando el extremo abdominal; gonapófisis con tres pares de valvas, las inferiores unidas a foliaciones basales, laminares, cuneiformes y visibles externamente. Bursa copulatrix con forma de un saco membranoso, su porción dorsobasal es de paredes engrosadas y se pliega formando dos divertículos. Espermateca simple, tubular, membranosa. Macho con placa subgenital cupuliforme, puntiaguda, con ápice truncado. Vómer esclerosado, delgado, puntiagudo y curvado dorsalmente. Pene asimétrico, constituido por un conjunto de unidades lobulares $L u 1,3,4$ y 5: un lóbulo mayor de disposición ventral forma un capuchón en su extremo distal ( $L u 1)$, en sus costados se acompaña de dos o tres lóbulos cortos (Lu1a, b y c); un lóbulo laminar de extremo dilatado se dispone al lado derecho (Lu3); al lado izquierdo se disponen dos lóbulos ( $L u 4)$ que portan en sus extremos estructuras para su mutuo anclaje, cuando están trabados configuran a todos los lóbulos de la genitalia en una estructura con forma de tubo; en el centro se despliega un lóbulo (Lu5) muy esclerosado con forma de varilla de ápice inflamada.

Huevo: Cara dorsal convexa, cara ventral recta; corion con retículos carenados con cerdas de longitud variable. Lámina micropilar ubicada en el extremo dorsal polar, proyectada hacia abajo por una prolongación del corion, que la separa del área polar. Opérculo circular con un alto capítulo en forma de corona, rugoso y con una cámara central. Ángulo opercular de $0^{\circ}$.

DistriBución: En Chile desde $33^{\circ} 55^{\prime} \mathrm{S}$; $70^{\circ} 57^{\prime} \mathrm{W}$ hasta los 35'49'S; 72³8’ W.

\section{Paraxeropsis camousseighti n. sp.}

(Figs. 1-8)

DESCRIPCIÓN DE IMAGOS:

Morfometría: Se señala en la Tabla 1. Habitus en las figuras 1 y 2.

Hembra (Figs. 1, 2a, 3-5): D e coloración parda amarillenta. Cabeza, tórax, abdomen y patas cubiertos por diminutos 
tubérculos y arrugas irregulares, sin foliaciones ni crestas (Figs. 1 y 2a). Cabeza oblonga, casi dos veces la longitud del pronoto, palpos maxilares y labiales aplanados. Antenas de largo similar al profémur, con escapo plano y ensanchado. Pronoto trapezoidal, más largo que ancho, de margen anterior cóncavo y posterior recto, deprimido medialmente. Mesonoto cilíndrico, dilatado en la inserción de las patas, cinco veces la longitud del pronoto. Metanoto de longitud similar al segmento mediado. Patas estriadas longitudinalmente, fémures con pequeñas espinas en los ángulos de articulación con la tibia, areola distotibial con un tubérculo prominente hacia los tarsos, fémures anteriores de corte transversal triangular, fémures medios y posteriores de corte transversal hexagonal, fémures medios con un pequeño lóbulo ennegrecido en el tercio proximal de la carena ventral interna. Abdomen con segmentos I a IX de margen posterior recto, primeros cinco segmentos subcilíndricos, los cinco distales comprimidos lateralmente (Fig. 3a), décimo tergo inclinado ventralmente (Fig. 3a superior), de margen posterior estrecho y bilobulado (asimétrico en el espécimen tipo) (Fig. 3a inferior). Placa subgenital, laminar, cóncava, progresivamente aguzada hacia el ápice, de ápice redondeado y sobrepasando ligeramente el margen del décimo tergo (Fig. 3a). Margen posterior del VII esterno con estrías laterales elevadas en torno a una abertura única y esclerosada (recibe la espina del vómer del macho) (Fig. 3b). Cercos (Fig. 3a) cortos, con forma de cono truncado inclinado, de ápice agudo, están cubiertos por el décimo tergo y sólo se expone lateralmente su ápice.

Tabla 1. Morfometría de Paraxeropsis camousseighti n. sp., longitudes en milímetros.

TABLE 1. Morphometry of Paraxeropsis camousseighti sp. n., lengths in millimeters.

\begin{tabular}{|c|c|c|}
\cline { 2 - 3 } \multicolumn{1}{c|}{} & え & + \\
\hline Largo total & 44,36 & 70,00 \\
\hline Cabeza & 3,21 & 3,57 \\
Antena & 26,40 & 21,4 \\
Pronoto & 2,50 & 3,21 \\
Mesonoto & 8,93 & 16,10 \\
Metanoto & 4,29 & 5,00 \\
\hline Seg. mediano & 2,50 & 5,00 \\
Seg. Abdominal II & 2,29 & 3,93 \\
Seg. Abdominal III & 2,86 & 4,29 \\
Seg. Abdominal IV & 2,86 & 4,57 \\
Seg. Abdominal V & 3,21 & 4,64 \\
Seg. Abdominal VI & 3,00 & 4,50 \\
Seg. Abdominal VII & 2,71 & 4,29 \\
Seg. Abdominal VIII & 2,00 & 4,14 \\
Seg. Abdominal IX & 2,21 & 4,29 \\
Seg. Abdominal X & 1,79 & 2,50 \\
Cerco & 1,29 & 0,43 \\
Placa subgenital & - & 12,10 \\
\hline Profémur & 17,50 & 21,4 \\
Protibia & 18,20 & 22,50 \\
Probasitarso & 4,43 & 3,57 \\
Total protarso & 7,14 & 6,43 \\
\hline Mesofémur & 12,40 & 15,00 \\
Mesotibia & 13,20 & 16,00 \\
Mesobasitarso & 2,86 & 2,50 \\
Total mesotarso & 5,00 & 5,00 \\
\hline Metafémur & 15,70 & 19,00 \\
Metatibia & 17,90 & 20,00 \\
\hline
\end{tabular}



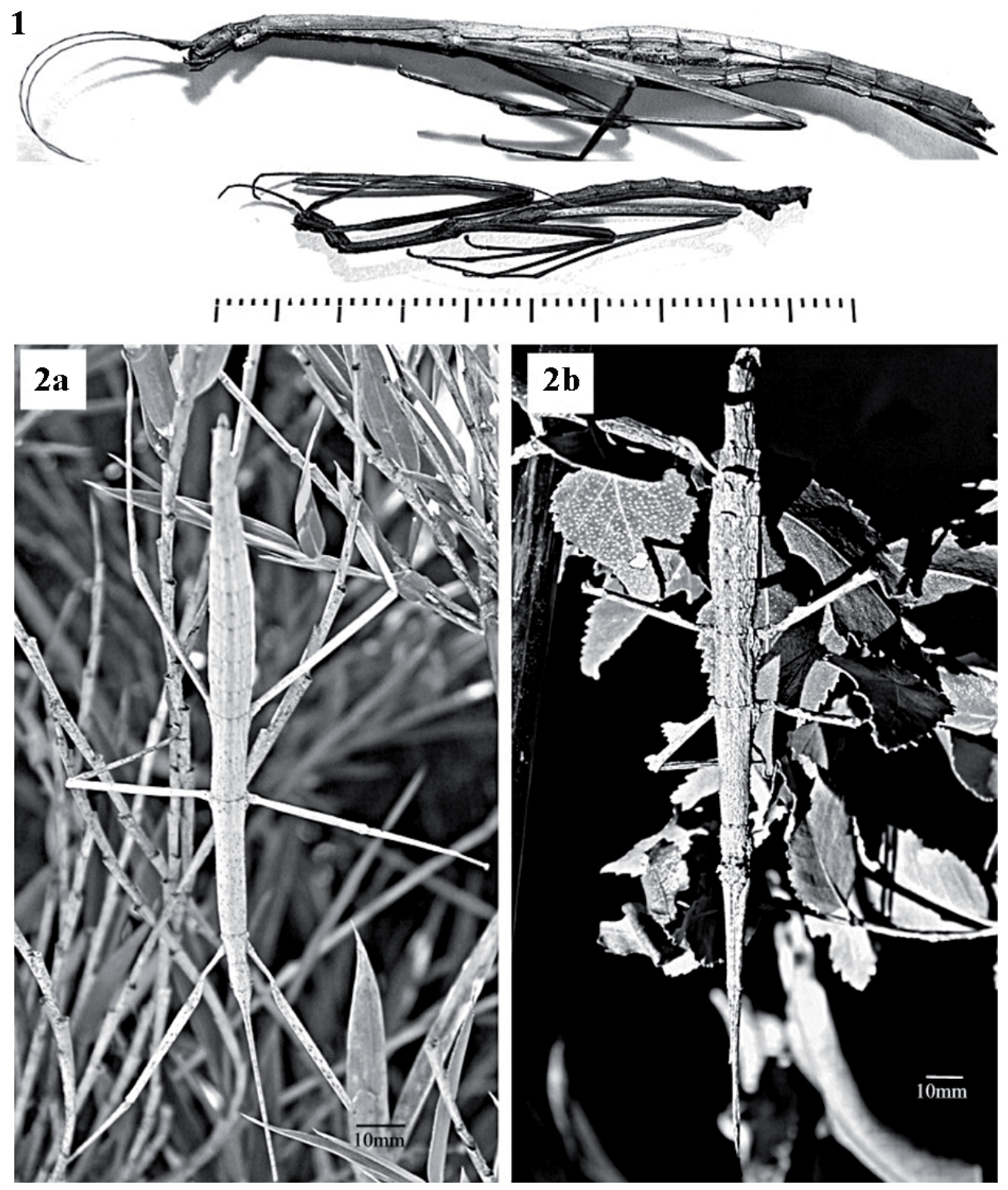

Figuras 1-2. 1, P. camousseighti imagos, $q$ superior y $d$ inferior, escala en milímetros. 2 , $q$ in vivo, a) $P$. camousseighti sobre Chusquea sp., b) P. bicristata sobre Nothofagus dombeyi.

Figures 1-2. 1, P. camousseighti images, $q$ top and $\hat{O}$ down, scale in millimeters. 2 , + in vivo, a) $P$. camousseighti on Chusquea sp., b) $P$. bicristata on Nothofagus dombeyi. 

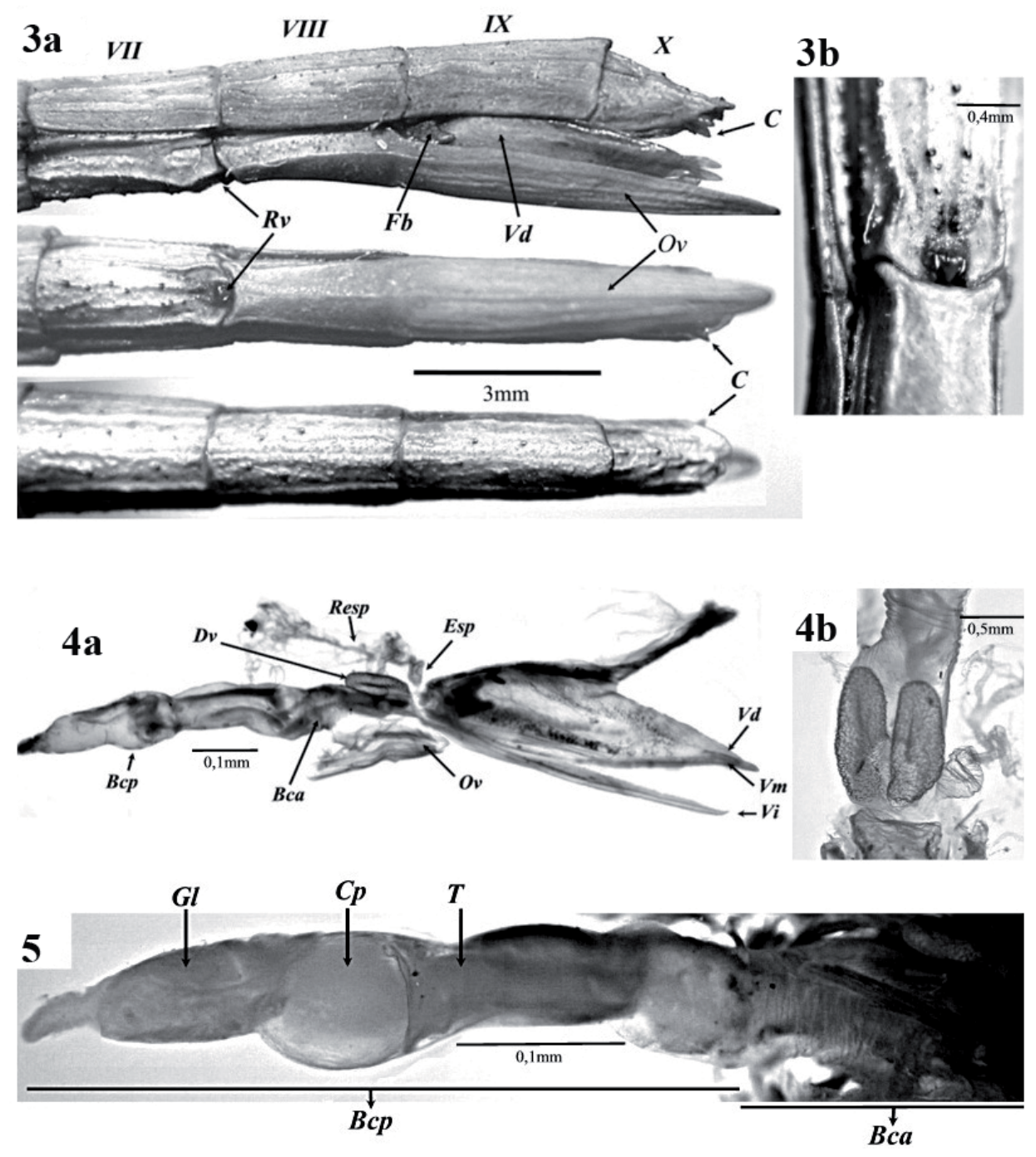

Figuras 3-5. 3 Abdomen de la hembra, a) lateral, ventral y dorsal, b) detalle del esterno VII. 4, abdomen disecado, a) lateral, b) dorsal, con divertículos y espermateca. 5, lateral, bursa copulatrix con espermatóforo.

Figures 3-5. 3 Female abdomen, a) lateral, ventral and dorsal, b) detail of sterno VII. 4, abdomen dissected, a) lateral, b) dorsal, with diverticula and spermateca. 5, lateral, bursa copulatrix with espermatofore. 
Gonapófisis (Fig. 4a) con forma de hojuelas, valva media y dorsal fundidas, sólo con los ápices separados, valva ventral libre con forma de cinta, con foliación basal cuneiforme, visible externamente (Fig. 3a). Bursa copulatrix como un saco agudo, que se extiende anteriormente hasta el sexto segmento abdominal (Figs. 4a y 5), con un par de divertículos más esclerosados, ubicados dorsalmente en el tercio anterior (Fig. 4a y b). Espermateca simple como un pequeño saco piriforme asociado a ramificaciones dorsales (Fig. $4 \mathrm{a}$ y b).

MACHo (Figs. 6-7): De color pardo oscuro, cuerpo cubierto con arrugas irregulares y tubérculos, los que pueden dar origen a espinas cortas asimétricamente distribuidas (Fig. 1 inferior). Cabeza oblonga semejante a la hembra, con bandas laterales de color negro que pasan por sobre los ojos, antenas similares a la hembra. Pronoto semejante al de la hembra, pero sin depresión medial, mesotórax cilíndrico, 3,6 veces el largo del pronoto, metanoto 1,7 veces el largo del Segmento mediado, ambos cilíndricos. Patas con estriación longitudinal, poco evidente, carenas y areolas como en la hembra, patas medias con carena ventral ondulada. Abdomen con segmentos I a VIII cilíndricos, ensanchados en torno a su articulación, de margen posterior recto con tubérculos alineados y prominentes (Figs. 1 y 6 ). Décimo tergo dirigido posteriormente (Fig. 6), de costados ensanchados en la zona de articulación de los cercos (Fig. 6c), margen posterior ensanchado y hendido, desarrollando dos lóbulos simétricos (Fig. 6c), la cara ventral de los lóbulos presentan espinas cortas (Fig. 7d). Placa subgenital cupuliforme, puntiaguda, con ápice truncado y bífido (Fig. 6a y b). Vómer esclerosado, delgado, puntiagudo y curvado dorsalmente (Fig. 7d). Cercos gruesos, cónicos, dirigidos ventralmente y de ápice curvado medialmente en ángulo recto (Figs. 6a, 7a y d).

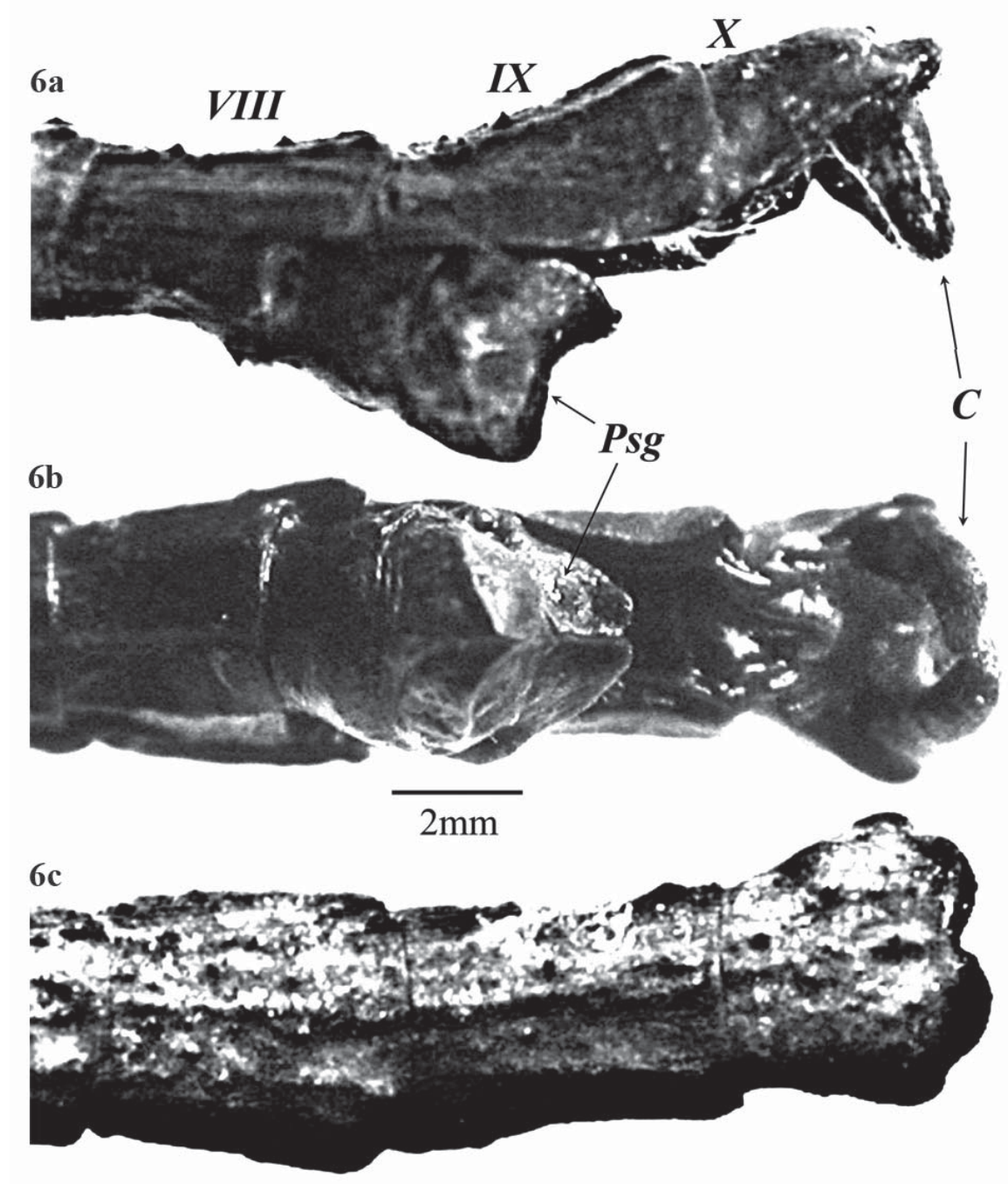

FIgURA 6. Extremo abdominal del macho en a) vista lateral, b) ventral y c) dorsal.

FIgURE 6. Apex of male abdomen in a) lateral, b) ventral and c) dorsal views. 
Pene asimétrico, constituido por un conjunto de lóbulos denominados aquí como: unidades 1, 3, 4 y 5. Lu1 representa el lóbulo de mayor tamaño, se despliega ventralmente hacia la derecha y forma un capuchón en su ápice (Fig. 7a, b y c), en sus costados se acompaña de otros lóbulos pequeños, Lu1a en la cara derecha, de forma globosa, $L u 1 b$ y $c$ en su costado dorsal, inclinados hacia la izquierda, ambos de tamaño pequeño, flexibles y digitiformes; Lu3 se constituye de un único lóbulo laminar cuyo extremo está dilatado y semeja una pesuña flexible (Fig. 7a, b y c), se dispone dorsalmente y rodea la base del pene de derecha a izquierda; Lu4 está formado por dos lóbulos laminares, anchos, que en su extremo presentan un mecanismo que los traba, se ubican ventral y al lado izquierdo, $L u 4 a$ porta una horquilla y $L u 4 b$ porta una masa trilobulada (Fig. 7a), cuando se traba la masa en la horquilla (Fig. 7c) el conjunto de lóbulos forma un tubo que desplaza hacia ventral la unidad Lu1; Lu5 es un vástago esclerosado de ápice plano y dilatado (Fig. 7a, b y c), éste se articula con una lámina esclerosada ubicada basalmente en el centro del pene.
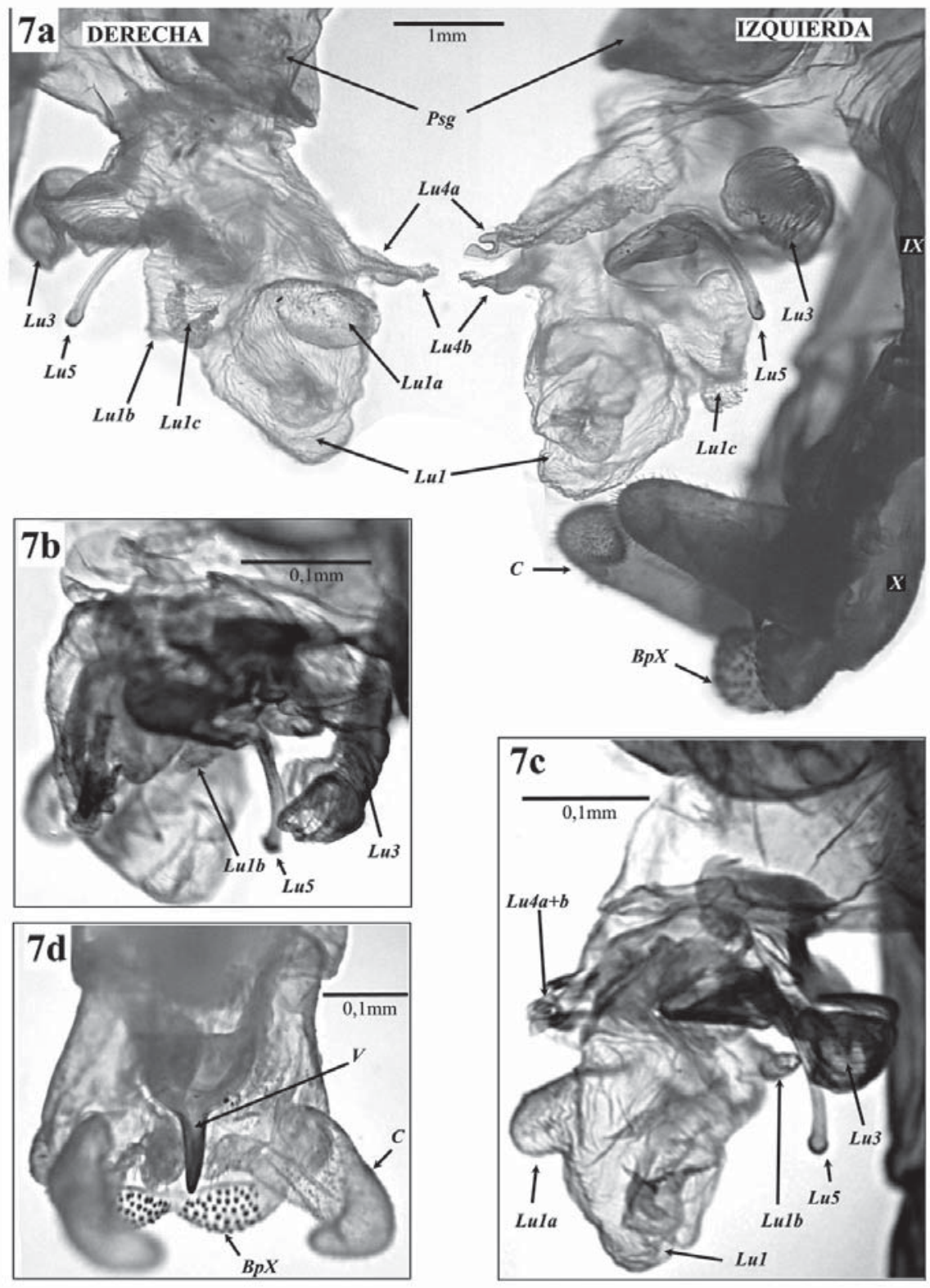

Figura 7a-d. Extremo abdominal del macho con los lóbulos genitales extendidos, a) vista lateral, derecha e izquierda, b) vista lateral izquierda con Lu3 extendido, c) vista lateral izquierda con Lu4a y b ensamblados, d) vómer en vista ventral.

Figure 7a-d. Apex of male abdomen with genital lobes exposes, a) lateral view, right and left, b) left lateral view with Lu3 exposes, c) left lateral views with Lu4a and b ensembles, d) vomer in ventral view. 
DESCRIPCIÓN DEL HUEVO (Fig. 8a-c):

Basado en 26 huevos. De forma alargada casi fusiforme, cara dorsal convexa, cara ventral recta, cápsula de corion ornamentado con retículos irregulares, definidos por carenas con cerdas diminutas (Fig. 8a). Lámina micropilar ubicada en el extremo dorsal inferior, proyectada en una prolongación del corion que la separa del área polar. Lámina con forma de "Lira" invertida, de copa micropilar amplia y dilatada ocupando todo el margen posterior de la lámina (Fig. 8c). Opérculo circular con capítulo en forma de corona cilíndrica, de diámetro similar al opérculo, de margen superior dentado, cada diente porta cerdas variables, el capítulo forma una cámara central (Fig. 8b). Ángulo opercular de $0^{\circ}$. Capítulo de $1 \mathrm{~mm}$ de largo, cápsula de $5 \mathrm{~mm}$ de largo, ancho de $1,4 \mathrm{~mm}$.
Material examinado: Holotipo: Imago $\widehat{\delta}^{\top}$ : Chile, Región

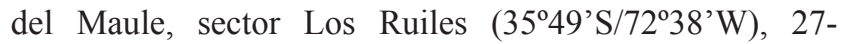
noviembre-2004, sobre Chusquea sp. y Blechnum chilense, col. A. Vera. Alotipo: Imago $q$ : mismos datos que el holotipo, se acompaña de huevos. Ambos depositados en la colección entomológica del Museo Nacional de Historia Natural, Santiago de Chile (MNHN).

Etimología: Camousseighti: especie dedicada al Dr. Ariel Camousseight, por su inmejorable contribución al conocimiento de los Phasmatodea de Chile y Argentina, con quien disfruté de apasionadas discusiones del tema. El Dr. Camousseight participó en la etapa inicial de este estudio hasta su fallecimiento el 13 de agosto de 2010.

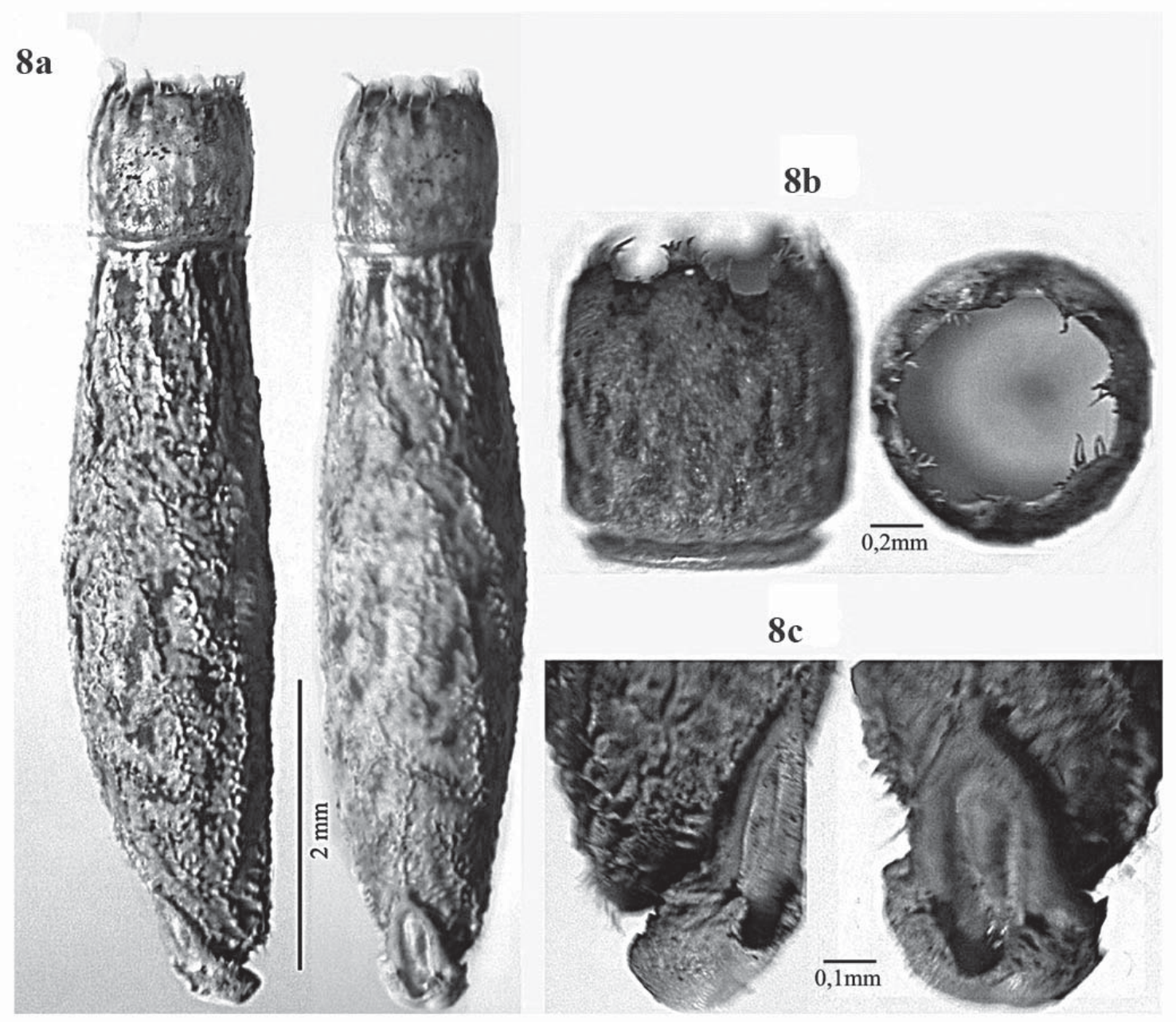

FigurAs 8a-c. Huevo, a) vista general, b) opérculo, c) lámina micropilar.

Figures 8a-c. Egg, a) general view, b) operculum, c) micropylar plate. 
OBSERVACIONES:

La hembra se encontraba en plena actividad de ovipostura cuando fue sacrificada, no presentaba restos externos del espermatóforo, pero al interior de la bursa copulatrix se encontraron elementos del espermatóforo y turgencia en los divertículos y la espermateca. La bursa contenía dos unidades repetidas (Fig. 5), cada una compuesta de un tubo esclerosado aplanado $(T)$, continuado de una cápsula esférica de color blanco-cristalino ( $C p)$ y en el extremo una glándula plegada de color blanco-cremoso $(G l)$. Estas estructuras son observadas por primera vez y no es posible interpretar su función. Puede tratarse de espermatóforos completos o restos de ellos. Las estrategias de transferencia espermática en los insectos pueden o no presentar espermatóforos, éstos exhiben variadas morfologías y pueden o no exponerse externamente en la genitalia femenina (Proctor 1998). Para Phasmatodea se han descrito espermatóforos con cápsulas externas (Bedford 1978, Clark 1975, Calberg 1981 y Camousseight \& Vera 2010), pero nunca como lo observado aquí. En cuanto a $P$. bicristata, no existen antecedentes al respecto.

\section{DISCUSIÓN}

P. camousseighti n. sp. se distingue claramente de P. bicristata. En su aspecto general, las hembras de P. camousseighti n. sp. carecen de las exageradas foliaciones en las patas, los segmentos abdominales y de las crestas cefálicas, mientras que los machos de ambas especies son más semejantes entre sí, ya que carecen de foliaciones, sin embargo los cercos de $P$. camousseighti n. sp. son cortos, robustos, de ápice curvado medialmente y están dirigidos ventralmente en ángulo recto, lo que contrasta con los cercos gráciles dirigidos posteriormente de $P$. bicristata. Ambas especies comparten importantes estructuras y patrones en los lóbulos de la genitalia masculina, que confirma su identidad congenérica, al tiempo que los distingue de Xeropsis como ya había sido señalado por Camousseight (2008), y de Splendidonemia, de esta última se distinguen porque su pene carece de la unidad 2 (Lu2) (Camousseight \& Vera 2011).

Camousseight (2008) describe los lóbulos fálicos para $P$. bicristata. Señala el mecanismo de la traba $(L u 4 a+b)$, donde la horquilla ( $L u 4 a)$ se ancla a una masa ( $L u 3$ ) en lóbulos opuestos. Tras reexaminar el espécimen tipo, fue posible identificar un error en la interpretación. La masa sobre Lu3 no participa en la traba, en su lugar lo hace el lóbulo $L u 4 b$, el que Camousseight describe como "un lóbulo puntiagudo al costado del capuchón”. Hecha esta enmienda, los lóbulos fálicos de ambas especies son homólogos, y se observan pocas diferencias específicas, como son: la ausencia de $L u 1 a$ en $P$. bicristata y el desarrollo en forma de pesuña de Lu3 en $P$. camousseighti n. sp.
En cuanto a la genitalia femenina, la organización de la bursa copulatrix es semejante en ambas especies y hasta ahora exclusiva del género. Ambas especies pueden distinguirse por la forma en que termina el décimo segmento abdominal y la placa subgenital, ambas estructuras se agudizan hacia el extremo distal en $P$. camousseighti n. sp., mientras que en $P$. bicristata no se agudizan y ambas estructuras terminan en un margen con amplios lóbulos distales.

En cuanto a los huevos, $P$. camousseighti muestra una morfología de la lámina micropilar y del opérculo sin precedentes, sin embargo ambas especies de Paraxeropsis coinciden en dos elementos básicos, el capítulo con forma de corona abovedada y la lámina micropilar proyectada desde el corion, más allá del margen polar.

Tal como había sido señalado por Camousseight (2005), los sectores cordilleranos costeros en Chile Central y Sur contienen una poco conocida fauna de Phasmatodea, que por sus asociaciones arborícolas pudo haberse sometido a fuertes perturbaciones, dada la actividad forestal de la región. $P$. camousseighti n. sp. presenta una acotada distribución en la Región del Maule, y pese a los extensos estudios y muestreos que se han desarrollado en estos bosques relictos, no había indicios de ella con anterioridad.

\section{AGRADECIMIENTOS}

A los árbitros que han contribuido a mejorar este trabajo, al Museo Nacional de Historia Natural, Entomología por facilitar laboratorios y equipos para realizar este estudio.

\section{BIBLIOGRAFÍA}

Bedford, G.O. 1978. Biology and Ecology of the Phasmatodea. Annual Review of Entomology 23:125-149.

BrunNer von Wattenwyl K. \& J. Redtenbacher. 1906-1908. Die Insekten familie der Phasmiden. Leipzig. 590 pp.

Calberg, U. 1981. Spermatophores of Baculum extradentatum (Brunner von Wattenwyl), and other Phasmida. Entomologist's monthly Magazine 117:125-127.

Camousseight, A. 1986. El género Xeropsis Redtenbacher, 1906 (Phasmatodea: Pseudophasmatidae). Revista Chilena de Entomología 14: 65-69.

Camousseight, A. 1988. Bacunculus cornutus comb. n. y B. blanchardi n.sp. de Chile (Insecta: Phasmatodea: Pseudophasmatidae.) Boletín del Museo Nacional de Historia Natural, Chile 41:117-123.

Camousseight, A. 2005. Recuadro 6. Los phasmidos de la Cordillera de la Costa. En: Historia, biodiversidad y ecología de los bosques costeros de Chile (Eds. SmithRamírez, C., J.J. Armesto \& C. Valdovinos), pp. 389-391. Editorial Universitaria. Santiago de Chile. 
Camousseight, A. 2008. Paraxeropsis bicristata n. gen y n. sp. de fásmido de Chile (Phasmatodea, Pseudophasmatidae). Boletín del Museo Nacional de Historia Natural, Chile 57: 109-117.

Camousseight, A. \& I. Bustamante. 1991. Descripción de los huevos de los fásmidos de Chile. Revista Chilena de Entomología 19:39-43.

Camousseight, A \& A. Vera. 2011. Redescripción de Splendidonemia splendida Zompro 2004 (Phasmatodea: Heteronemiidae), con descripción de la hembra y el huevo. Revista Chilena de Entomología 36: (en prensa).

Clark, J.T. 1975. A Conspicuous spermatophore in the phasmid Extatosoma tiaratum Macleay. Entomologist's monthly Magazine 110:81-82.

GraY, G.R. 1835. Synopsis of the species of insects belonging to the family of Phasmidae. Longman, Rees, Orme, Brown, Green, and Longman, Paternoster Row. London. 48 pp.

Proctor, H.C. 1998. Indirect Sperm Transfer in Arthropods: Behavioral and Evolutionary Trends. Annual Review of Entomology 43:153-174.

SELLICK, J.T.C. 1997. Descriptive terminology of the phasmid egg capsule, with an extended key to the phasmid genera based on egg structure. Systematic Entomology 22: 97-122.

SelLick, J.T.C. 2000. The eggs of some Chilean Phasmida (Phasmida: Pseudophasmatidae). Phasmid Studies 7(1): 1-5.

Zompro, O. 2004. Revision of the genera of the Areolatae, including the status of Timema and Agathemera (Insecta, Phasmatodea). Abhandlungen des Naturwissenschaftlichen Vereins in Hamburg (NF) 37: 3-327.

Recibido: 16.09 .10

Aceptado: 30.03.11 